\title{
Research on Fault Feature Extraction Method of Rolling Bearing Based on NMD and Wavelet Threshold Denoising
}

\author{
Maohua Xiao $\mathbb{D D}^{1,2}$ Kai Wen, ${ }^{1}$ Cunyi Zhang, ${ }^{1}$ Xiao Zhao, ${ }^{2}$ Weihua Wei, ${ }^{3}$ and Dan Wu ${ }^{1}$ \\ ${ }^{1}$ College of Engineering, Nanjing Agriculture University, Nanjing 210031, China \\ ${ }^{2}$ Faculty of Engineering and the Environment, University of Southampton, Southampton SO17 1BJ, UK \\ ${ }^{3}$ College of Mechanical and Electronic Engineering, Nanjing Forestry University, Nanjing 210037, China
}

Correspondence should be addressed to Maohua Xiao; xiaomaohua@njau.edu.cn

Received 28 February 2018; Revised 7 June 2018; Accepted 3 July 2018; Published 19 August 2018

Academic Editor: Carlo Rainieri

Copyright (C) 2018 Maohua Xiao et al. This is an open access article distributed under the Creative Commons Attribution License, which permits unrestricted use, distribution, and reproduction in any medium, provided the original work is properly cited.

Rolling bearings are the core components of the machine. In order to save costs and prevent accidents caused by bearing failures, the rolling bearing fault diagnosis technology has been widely used in the industrial field. At present, the proposed methods include wavelet transform, morphological filtering, empirical mode decomposition (EMD), and ensemble empirical mode decomposition (EEMD), which have obvious shortcomings. As it is difficult to extract the fault characteristic frequency caused by nonlinear and nonstationary features of the rolling bearing fault signal, this paper presents a fault feature extraction method of rolling bearing based on nonlinear mode decomposition (NMD) and wavelet threshold denoised method. First of all, the fault signal was preprocessed via wavelet threshold denoising. Then, the denoised signal was decomposed by using NMD. Next, the mode component envelope spectrum was made. Finally, the fault characteristic frequency of rolling bearing was extracted. The method was compared with EMD through the simulation experiment and rolling bearing fault experiment. Meanwhile, two indicators including signal-noise ratio (SNR) and root-mean-square error (RMSE) were also established to evaluate the fault diagnosis ability of this method, and the results show that this method can extract the fault characteristic frequency accurately.

\section{Introduction}

Rolling bearings are called "industrial joints." Bearing industry, the basic and key industry of machinery industry, often represents or restricts the development level of a country's machinery and other related industries. However, poor working conditions often affect their health status [1]. In order to save costs and prevent accidents due to bearing failures, the rolling bearing fault diagnosis technology has been widely used in the industrial field [2] Rolling bearing failure and complex interference signals, including pulse and modulation signals, likely affect the extraction of fault characteristic frequency. Signals from complex systems usually consist of a mixture of different oscillations, so the signals should be decomposed to make reliable analysis, namely, to restore the various oscillations present therein, separating them from each other and from the presence of noise interference [3]. How to effectively extract the fault characteristic frequency is a key technical problem to bearing fault diagnosis.

The proposed methods include wavelet transform, morphological filtering, Hilbert-Huang transform, empirical mode decomposition (EMD), and ensemble empirical mode decomposition (EEMD) [4], in which obvious shortcomings could be found. For example, the wavelet transform can effectively suppress white noise, but it is unable to suppress the interference of the pulse signal completely [5]. Although the morphological filtering can effectively suppress impulsive interference ability, the algorithm is simple and feasible [6], but there are some problems such as statistics offset and element selection [7]. As for the empirical mode decomposition [8], it does not have good antinoise [9]; moreover, low efficient algorithms [10], modal aliasing, and other issues [11] remain unsolved. In order to reduce the sensitivity of EMD to noise, $\mathrm{Wu}$ and Huang proposed a variant called ensemble empirical mode 
decomposition (EEMD) [12], which added the white Gaussian noise to the signal using the EMD method [13]. However, EEMD cannot be a good antinoise method [14].

Therefore, these methods have the following deficiencies:

(1) The method contains user-defined parameters and is very sensitive to the choice of parameters. These parameters cannot be adaptively selected, and there is no more or less common setting.

(2) The method does not have good noise robustness.

(3) If a single mode has a complex (nonsinusoidal) waveform, the method breaks it down into several oscillations with simpler waveforms [15].

(4) The modes returned by some methods are not always physically meaningful. For example, some methods even decompose a random signal (such as Brown noise) into a set of oscillations [15].

In view of these problems mentioned above, this paper presents a fault feature extraction method based on nonlinear mode decomposition and wavelet threshold denoising, which can solve these problems. Firstly, the signal is preprocessed by using wavelet threshold denoising, and then the signal of noise reduction is processed by nonlinear mode decomposition. The corresponding time domain and envelope spectra are drawn and analyzed to obtain the fault characteristic frequency of the rolling bearing.

\section{Wavelet Threshold Denoising}

Analysis of the vibration signal found that the useful signal was often a low-frequency or a relatively stable signal, while the noise signal was usually a high-frequency one [16]. As a multiscale signal analysis method, wavelet transform has a certain capability of recognition in the time-frequency domain. The improvement of the Fourier transform has powerful data analysis capability and perfect theoretical basis [17]. In the mechanical fault diagnosis, wavelet threshold denoising has been widely used for its excellent denoising and high-computational efficiency [18]. The basic idea of wavelet threshold denoising is to decompose the original signal by wavelet decomposition [19]. First, filter the coefficients after decomposition, and divide the coefficients into larger or smaller than a specific value. Then, reconstruct the selected components to obtain a preprocessed signal. Wavelet threshold denoising includes hard threshold denoising and soft threshold denoising [20]. The hard threshold denoising may preserve local features such as the edge of the signal, while the soft threshold denoising signal is relatively smooth [21].

(1) The hard threshold denoising function is defined as

$$
f(\lambda)= \begin{cases}0, & |\lambda|<\delta \\ \lambda, & |\lambda| \geq \delta\end{cases}
$$

where $\lambda$ and $\delta$ denote the wavelet coefficients and the given threshold, respectively. $f(\lambda)$ is the wavelet coefficient after applying the threshold [22].
(2) The soft threshold denoising function is defined as

$$
f(\lambda)= \begin{cases}0, & |\lambda|<\delta, \\ \operatorname{sign}(\lambda)(|\lambda-\delta|), & |\lambda| \geq \delta,\end{cases}
$$

where sign means the function of obtaining a value [23].

The acquisition of thresholds is often achieved by estimation. Three commonly used threshold estimation methods are as follows [24]:

(1) General threshold:

$$
\delta_{1}=\sigma \sqrt{2 \lg N},
$$

where $\delta_{1}, N$, and $\sigma$ denote the given threshold, the length of wavelet coefficients, and standard deviation of noise, respectively.

(2) Adaptive threshold selection based on unbiased likelihood estimation principle:

$$
\delta_{2}=\sigma \sqrt{\omega_{\mathrm{b}}},
$$

where $\delta_{2}, \omega_{\mathrm{b}}$, and $\sigma$ denote the given threshold, risk function, and standard deviation of noise, respectively.

(3) Heuristic optimal threshold estimation:

This is the synthesis of the first two methods and is the optimal predictor of threshold selection. When the signal to noise ratio is large, use the general threshold; when the signal to noise ratio is small, use unbiased likelihood estimation of the threshold. Let the sum of squares of $n$ wavelet coefficients be $s$ :

$$
\begin{aligned}
& \nu=\frac{(s-n)}{n}, \\
& \omega=\left(\log _{2} n\right)^{3 / 2} \sqrt{n} .
\end{aligned}
$$

The threshold value is

$$
\delta_{3}= \begin{cases}\delta_{1}, & v<\omega, \\ \min \left(\delta_{1}, \delta_{2}\right), & \nu>\omega .\end{cases}
$$

\section{NMD}

3.1. Basic Principle of NMD. Nonlinear mode decomposition (NMD) is an adaptive decomposition tool for any waveform, decomposing a given signal into a set of actually needed and meaningful oscillations while excluding noise. NMD is a powerful combination based on time-frequency analysis techniques, and its adaptive selection of parameters makes it extremely noise robust; surrogate data tests are used to identify interdependent oscillations and to distinguish deterministic from random activity [15].

The main goal of NMD is to decompose a given signal into a set of nonlinear modes, which is defined as the sum of all the components corresponding to the same activity [15]. The NMD principle is as follows. 
First of all, according to the selected resolution parameters $f_{0}$ ( $f_{0}$ is the resolution parameter determining the relationship between time and frequency resolution, namely, can reflect how fast the time changes and solve how close in the frequency components, by default $f_{0}=1$ ), use the wavelet formula to calculate the WT (wavelet transform) of a given signal. The formulas are as follows:

$$
\begin{aligned}
W_{s}(\omega, t) & \equiv \int_{-\infty}^{\infty} s^{+}(u) \psi^{*}\left[\frac{\omega(u-t)}{\omega_{\psi}}\right] \frac{\omega d u}{\omega_{\psi}} \\
& =\frac{1}{2 \pi} \int_{0}^{\infty} e^{i \xi t} \widehat{s}(\xi) \widehat{\psi}^{*}\left(\frac{\omega_{\psi} \xi}{\omega}\right) d \xi,
\end{aligned}
$$

where

$$
\begin{aligned}
\widehat{\psi}(\xi) & =e^{\left.-\left(2 \pi f_{0} \ln \xi\right)^{2}\right) / 2}, \\
\omega_{\psi} & =1 .
\end{aligned}
$$

Second, the principal component is extracted from the WT of the signal (defined as a reference component) and reconstructed. The principal component reconstruction formulas are as follows:

$$
\begin{gathered}
\nu(t)=\omega_{\mathrm{p}}(t) e^{\delta \ln v_{\mathrm{d}}(t)}, \\
A(t) e^{i \phi(t)}=\frac{2 W_{\mathrm{s}}\left(\omega_{\mathrm{p}}(t), t\right)}{\widehat{\psi}^{*}\left[\omega_{\psi} \nu(t) / \omega_{\mathrm{p}}(t)\right]},
\end{gathered}
$$

where $\omega_{\mathrm{p}}(t)$ is defined as the amplitude peak sequence after the WT transformation.

The reference component is then noise tested using surrogates. If it does not pass this test, stop the decomposition. Then, check if WT is the "correct" representation of the reference component, and if not, switch to using the WFT (windowed Fourier transform) and reextract the component from it. The formulas are as follows:

$$
\begin{aligned}
& S<1 \Rightarrow \text { use } \quad \text { WFT, } \\
& S>1 \Rightarrow \text { use } \quad \text { WT, } \\
& S \equiv\left\{1+V\left[\partial_{\mathrm{t}} \nu(t), \nu(t)\right]\right\}^{-1}+\left\{1+V\left[\partial_{\mathrm{t}} \nu(t), \nu(t)\right]\right\}^{-1},
\end{aligned}
$$

where

$$
V[m(t), n(t)] \equiv \frac{\operatorname{std}\left[|m(t) / \mathrm{n}(\mathrm{t})|^{+}\right]}{\operatorname{std}\left[|m(t) / \mathrm{n}(\mathrm{t})|^{+}\right]}
$$

where

$$
\begin{aligned}
|m(t)|^{+} & \equiv\left|m^{+}(t)\right|, \\
m^{+}(t) & \equiv \int_{0^{+}}^{\infty} \widehat{m}(\xi) e^{i \xi t} d \xi, \\
m(t) & =\frac{1}{T} \int m(t) d t, \\
\operatorname{std}[m(t) & \left.\equiv \sqrt{[m(t)]^{2}-[m(t)]^{2}}\right] .
\end{aligned}
$$

In the following, TFR means the selected representation: WT or WFT. For $h$ (harmonic) $=1 / 2,1 / 3, \ldots$, do the following:

(a) Calculate the time-frequency representation (TFR) of the signal over a defined frequency range using different values of the resolution parameter $f_{0}^{(h)}$.

(i) Extract the $h$ th harmonic of the reference component from the TFR and reconstruct its amplitude, phase, and frequency as follows:

$$
\begin{aligned}
A^{(h)} & =a_{h} A^{(1)}(t), \\
\phi^{(h)}-h \phi^{(1)} & =\varphi_{h}, \\
\nu^{(h)}(t) & =h \nu^{(1)}(t) .
\end{aligned}
$$

(ii) Test whether the current harmonic is true.

(b) If for some $f_{0}^{(h)}$ the harmonic is determined to be true, then set its characteristics to those reconstructed for $f_{0}^{(h)}$ that is characterized by the highest consistency with the reference component among $f_{0}^{(h)}$ for which the harmonic is determined to be true.

(c) Stop when a prechosen number (default =3) of consequent harmonics are determined to be false for all tested $f_{0}^{(h)}$.

If you have identified some harmonics as true in the previous step, use the true harmonic with the smallest $h$ as the reference component. Therefore, the reference component is guaranteed to be the first harmonic of the corresponding NM now. Perform the above steps from (a) to (c) for $h=1,2, \ldots$, remembering the reconstructed harmonic parameters. Then, reconstruct the complete NM using the harmonic parameters determined to be true in the previous step. Finally, subtract the reconstructed NM from the signal and repeat all the above steps for the residual.

The above is the basic decomposition principle of NMD. The specific decomposition method of NMD can be summarized in the following steps:

(a) Accurately extract the fundamental harmonic of NM from the TFR of the signal

(b) Find all possible candidates for harmonics based on their properties

(c) Identify the true harmonics (relative to the same $\mathrm{NM}$ ) among them

(d) Subtract the resulting NM from the signal (obtained by combining all the true harmonics), and iterate the procedure on the residual until the preset stop criteria are met.

3.2. Analog Signal Comparison. In order to verify the effectiveness of the NMD method, the following signal is used for simulation experiment: 


$$
\begin{aligned}
s(t)= & A[\cos (x)+0.5 \cos (3 x-\pi / 4)+0.33 \cos (4 x+\pi / 2) \\
& +0.25 \cos (7 x+\pi / 3)]+m,
\end{aligned}
$$

where

$$
\begin{aligned}
& A=1+0.25 \cos (2 \pi t / 20), \\
& x=2 \pi t+0.5 \sin (2 \pi t / 6),
\end{aligned}
$$

where $m$ is a random noise signal and its amplitude is one. The original signal, the noise-added signal, and the signal decomposed by various decomposition methods are shown in Figure 1.

As can be seen from the three graphs in Figure 1, the nonlinear mode decomposition (NMD) method can effectively eliminate the noise and extract the original signal from the complex mixed signal. The EMD and EEMD methods are used to decompose the signals added to the noise as shown in Figures 2 and 3, respectively.

Figure 2 is obtained by using the EMD method to decompose the signal added to the noise, while Figure 3 uses the EEMD method to decompose the signals added to the noise. Unlike the NMD method, the EMD method produces 13 different components, of which only 4th components extract the original signal approximately, and only 5th components approximately extract the original signal in the EEMD decomposed one. The other components produced by EMD and EEMD either have noise mixing or signal corruption.

\section{Fault Feature Extraction of Rolling Bearing}

Rolling bearing fault feature extraction process is shown in Figure 4.

Specific steps are as follows:

(1) The original signal is processed by wavelet threshold to obtain the preprocessed noise reduction signal.

(2) Perform NMD decomposition on the noise-reduced signal to obtain a new component.

(3) Calculate the cross-correlation coefficient of the component and the original signal.

(4) Draw the envelope spectrum of the component.

(5) Determine in the envelope spectrum whether a fault has occurred and where the fault occurred.

\section{Application of Fault Extraction of Rolling Bearing}

Conducting the research and analysis of the example of the rolling bearing, the methods of NMD and EMD are used, respectively, to deal with the faulty vibration signals generated in the bearing outer ring and the inner ring to prove the effectiveness of the method [25].

Formula of the inner race frequency:

$$
f_{\mathrm{i}}=\frac{1}{2}\left(1+\frac{d}{D} \cos \alpha\right) f_{n} Z .
$$

Formula of the outer race frequency:

$$
f_{\mathrm{o}}=\frac{1}{2}\left(1-\frac{d}{D} \cos \alpha\right) f_{n} Z,
$$

where $d$ and $D$ denote the ball diameter and the pitch diameter, respectively. $Z$ is the number of rolling elements, $\alpha$ denotes the bearing contact angle, and $f_{n}$ is the rotation frequency [26].

To further verify the proposed method, we used the real motor bearing data provided by Case Western Reserve University. Vibration data were picked up with a sampling frequency of $12 \mathrm{kHz}$ by an accelerometer placed at the 6 o'clock position at the drive end of the motor housing. Single point faults were processed in the bearings using electrodischarge machining with a fault diameter of 0.021 inches, and the fault depth was 0.011 inches. In addition, the motor speed was $1797 \mathrm{rpm}$. The characteristic frequencies of the bearing are shown in Table 1, which were calculated by formulas (15) and (16).

The raw inner race vibration signal and its spectrum are shown in Figure 5. And the time domain map and spectrum of the inner ring fault signal after wavelet threshold denoising are shown in Figure 6. As can be seen from Figure 5, due to the signal mixed with serious noise, it is difficult to identify the fault characteristic frequency making the spectrum of high-frequency components increase.

After being decomposed by NMD and EMD, respectively, the cross-correlation coefficient between the decomposed components and the original signal is calculated and the envelope spectrum is drawn. Usually, the crosscorrelation coefficient of the first two layers is large. We set a threshold of 0.1 here and use the IMF with a coefficient greater than 0.1 for reconstruction to draw the envelope spectrum. In the selection of wavelet basis function decomposition, the experiment proved $\mathrm{db} 10$ wavelet decomposition signal SNR is better. In order to ensure that experiments are conducted under the same conditions, the "db10" wavelet decomposition of the original signal is performed. Since the NMD method has an adaptive feature, it does not need parameter settings.

Figures 7 and 8 are the envelope spectra of inner ring fault signals, respectively, after wavelet threshold denoising based on NMD and EMD. Through comparison, the extraction effect in Figure 7 is obviously better than that in Figure 8. In Figure 7, we can accurately extract rotation frequency, fault characteristic frequency, and frequency multiplication thereof, thus verifying the effectiveness of the method.

Further analysis is conducted on the fault signal of the bearing outer ring, and the raw outer race vibration signal and its spectrum are shown in Figure 9. The time domain map and spectrum of the outer ring fault signal after wavelet threshold denoising are also shown in Figure 10.

After the denoising by wavelet threshold, NMD and EMD are, respectively, used to decompose them to calculate the cross-correlation coefficient between the decomposed components and the original signal to draw the envelope spectrum. 


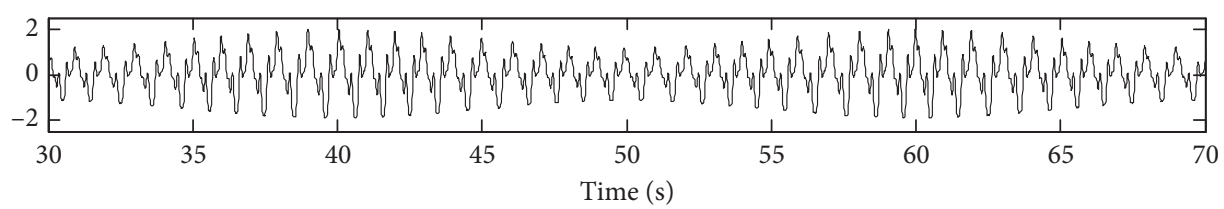

(a)

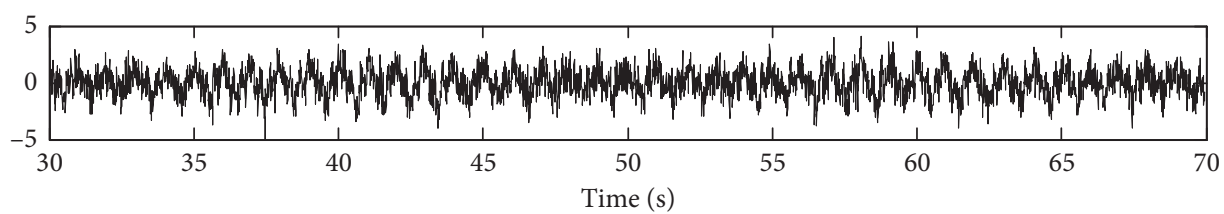

(b)

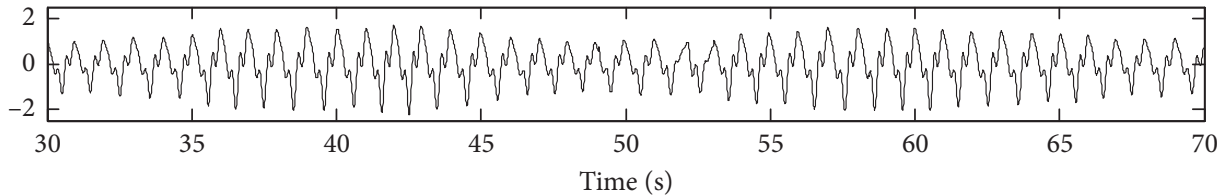

(c)

Figure 1: (a) Original signal. (b) Noise-added signal. (c) Signal extracted by NMD.
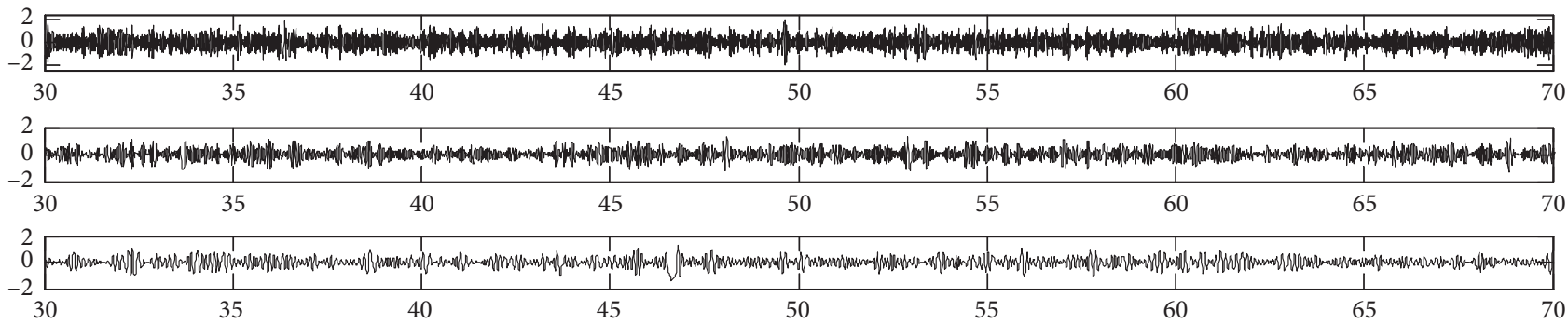

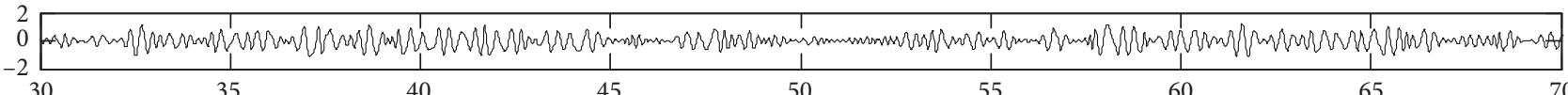

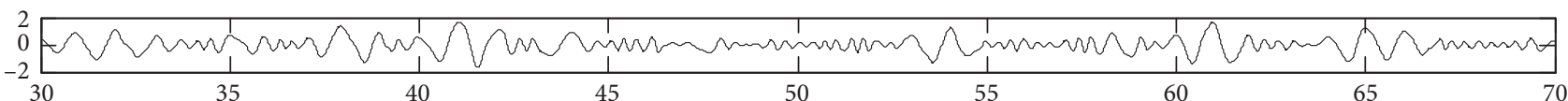

$\mathrm{H}_{30}^{2}$
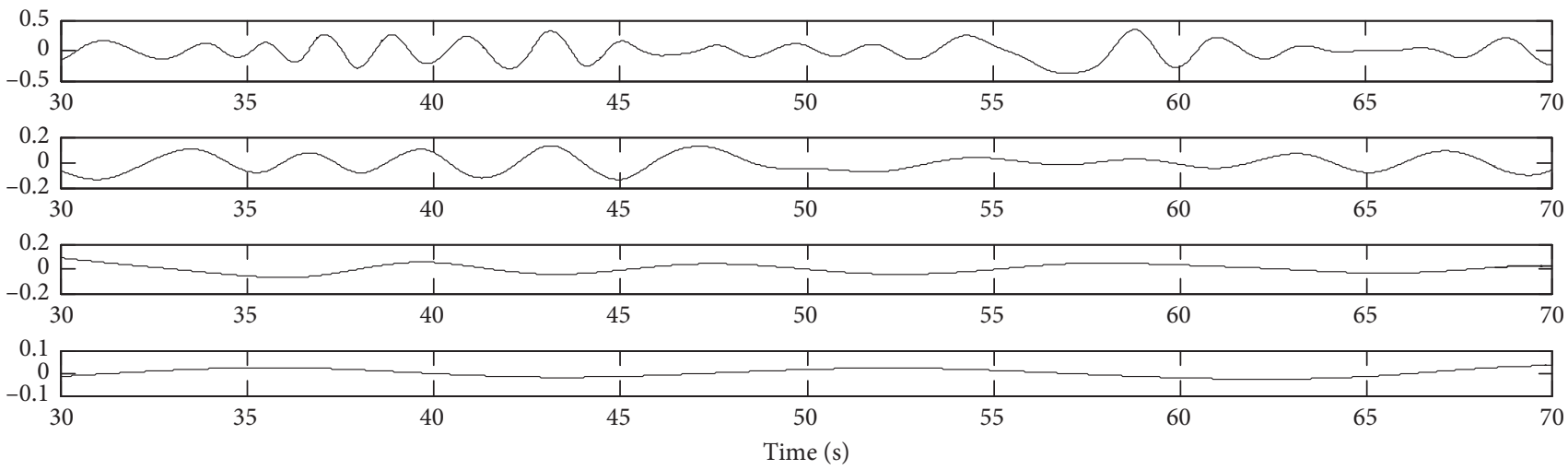

Figure 2: Signal extracted by EMD. 

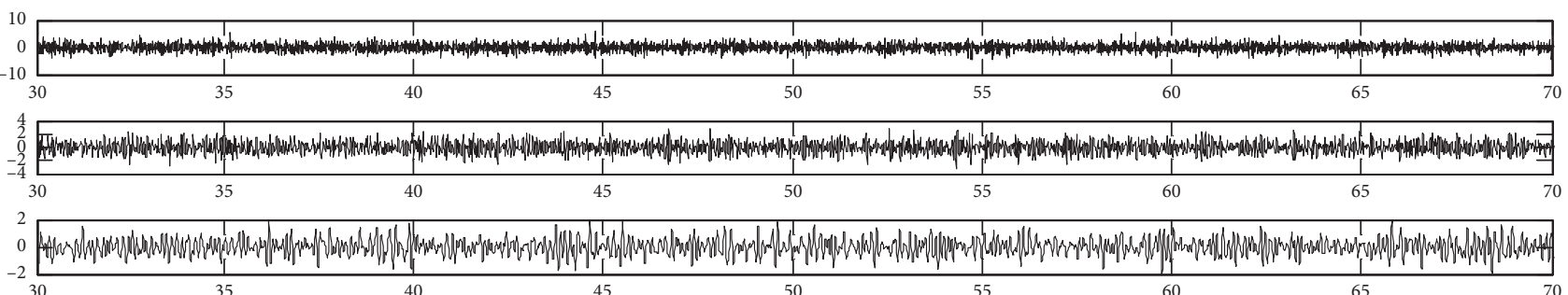

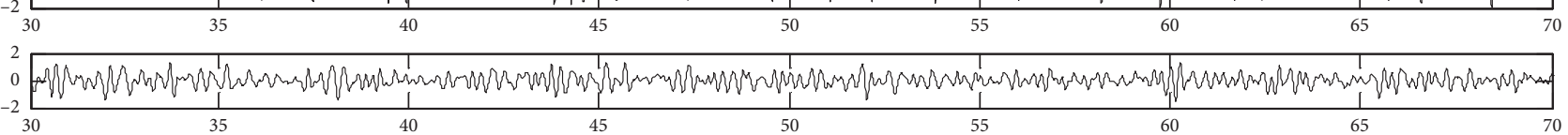

${ }_{30}^{2}$

10
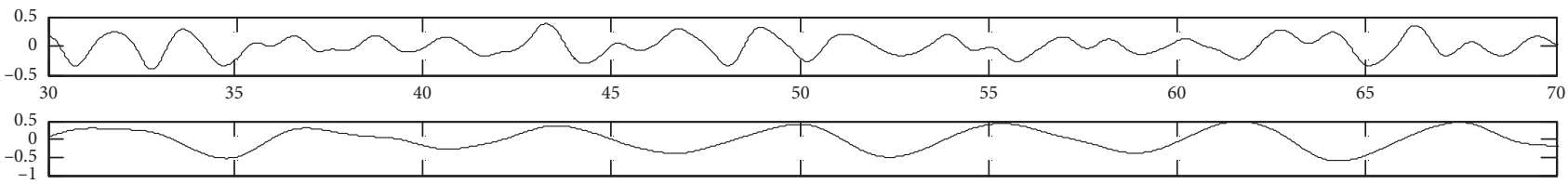

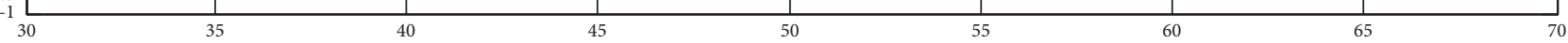

(10.2)

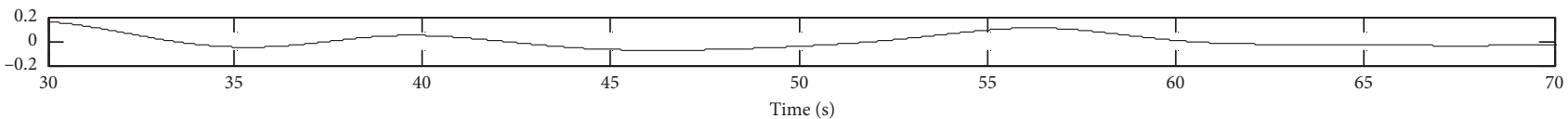

Figure 3: Signal extracted by EEMD.

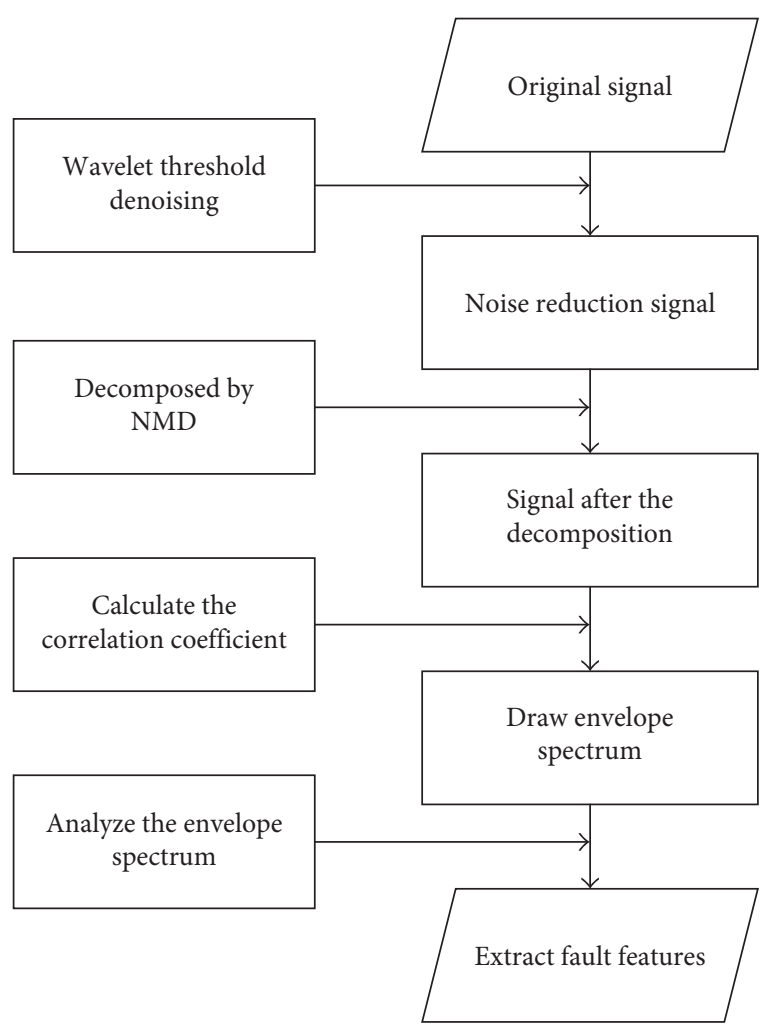

FIGURE 4: Fault feature extraction flow diagram.
TABLE 1: Fault characteristic frequencies of bearing. Shaft frequency, Outer race frequency, Inner race frequency, $\begin{array}{lll}f_{n} & f_{\mathrm{o}} & f_{\mathrm{i}}\end{array}$ $29.32 \mathrm{~Hz}$

$107.32 \mathrm{~Hz}$

$162.29 \mathrm{~Hz}$

Figures 11 and 12 show the result of wavelet threshold denoising based on NMD and EMD for the outer ring fault signal, respectively. It can be seen from the comparison that the extraction effect in Figure 11 is slightly better than that in Figure 12. Although the advantages of NMD in these two figures are not obvious, the calculation of the SNR formula (19) and the RMSE formula (20) can result in a larger signalnoise ratio and a smaller root-mean-square error for the NMD method. And the algorithm has a shorter processing time and higher diagnostic efficiency. And the NMD algorithm needs to perform fewer basic operations than the EMD algorithm.

Through formula (17), respectively, calculate their crosscorrelation coefficient as follows [27]:

$$
r_{X Y}=\frac{\sum_{i=1}^{N}\left(X_{i}-\bar{X}\right)\left(Y_{i}-\bar{Y}\right)}{\sqrt{\sum_{i=1}^{N}\left(X_{i}-\bar{X}\right)^{2}} \sqrt{\sum_{i=1}^{N}\left(Y_{i}-\bar{Y}\right)^{2}}} .
$$

It can be seen from Figure 13 that the cross-correlation coefficient of the inner ring fault signal after the wavelet threshold denoising and NMD is 0.8788 and the 


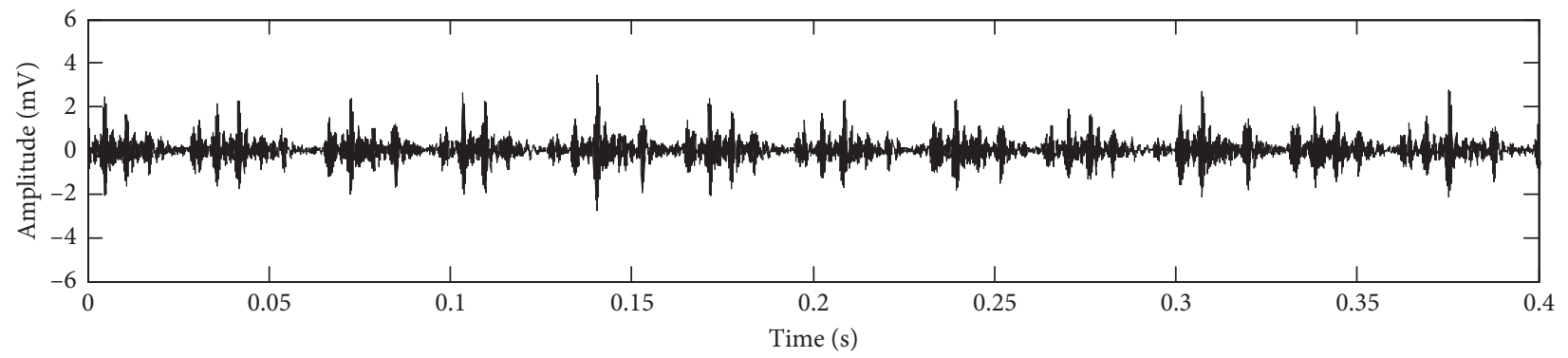

(a)

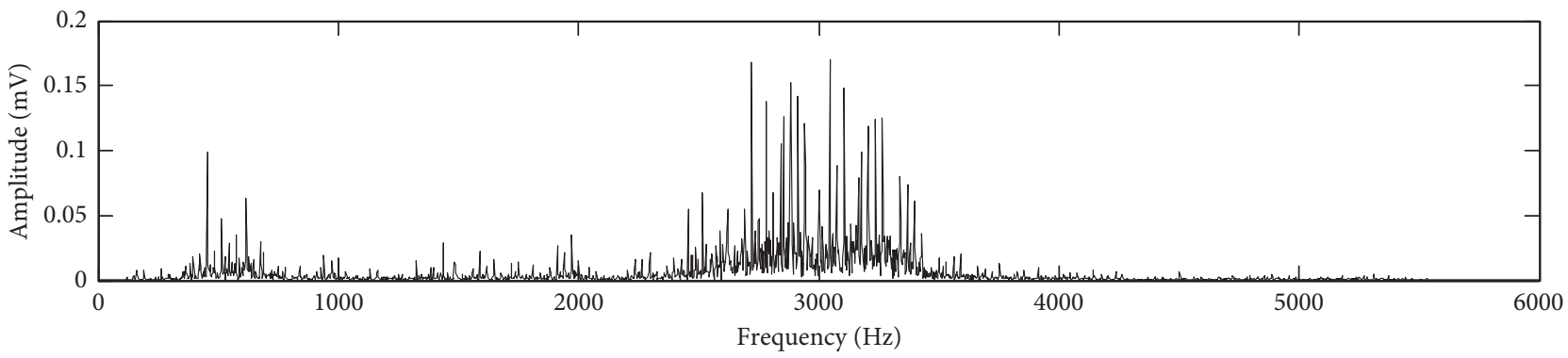

(b)

FIGURE 5: The inner ring fault signal time domain map and its spectrum.

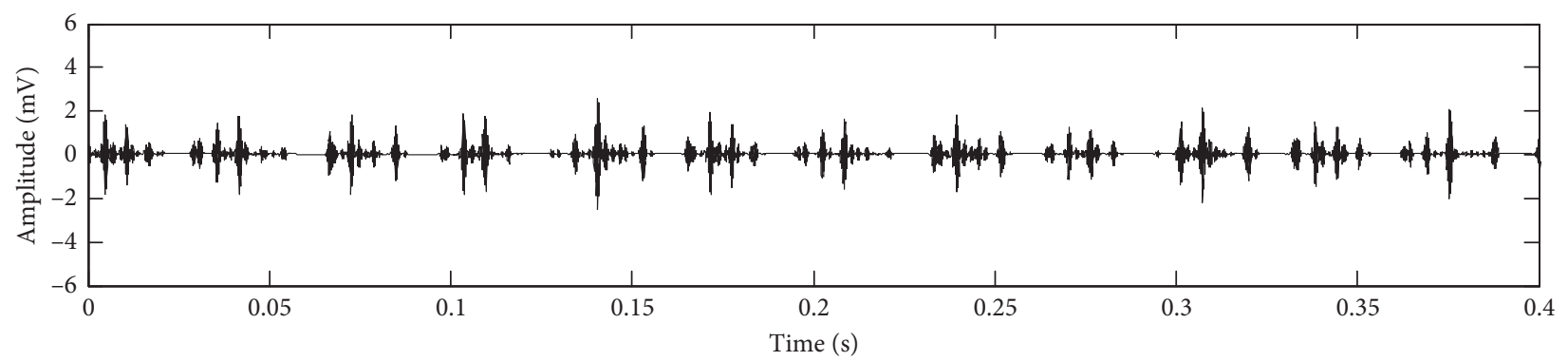

(a)

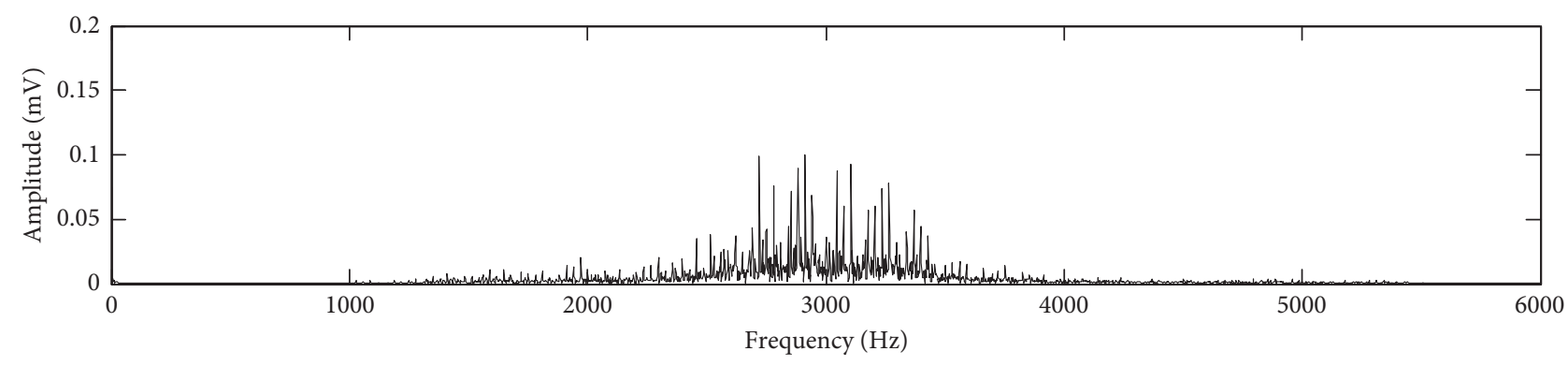

(b)

FIgURE 6: The inner ring fault signal time domain map and its spectrum after wavelet threshold denoising.

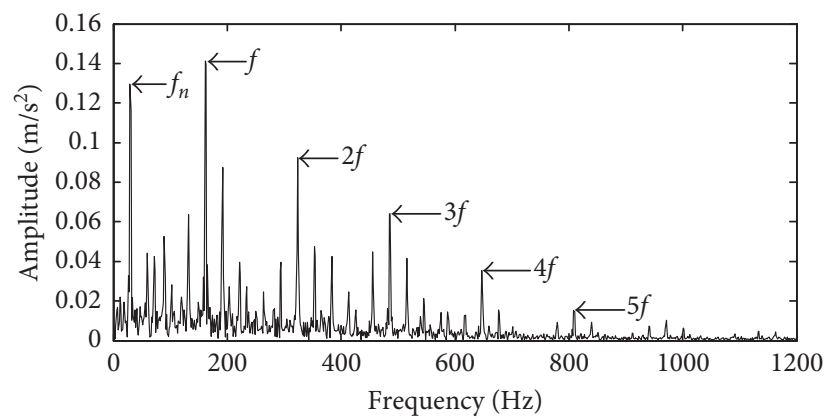

FIGURE 7: Envelope spectrum after NMD of the inner ring fault signal. 


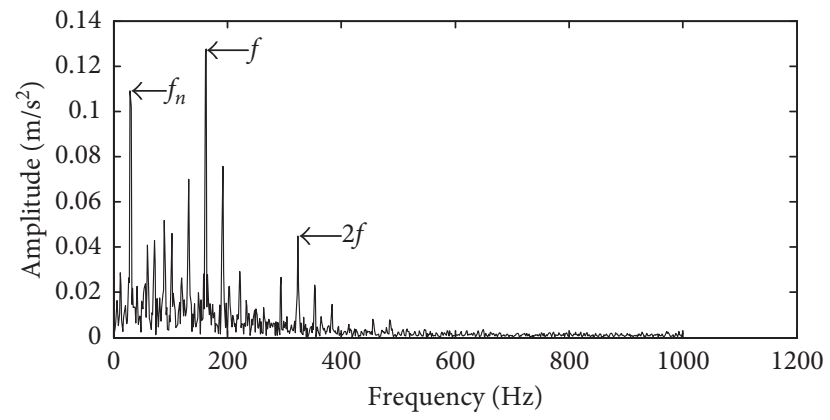

Figure 8: Envelope spectrum after EMD of the inner ring fault signal.

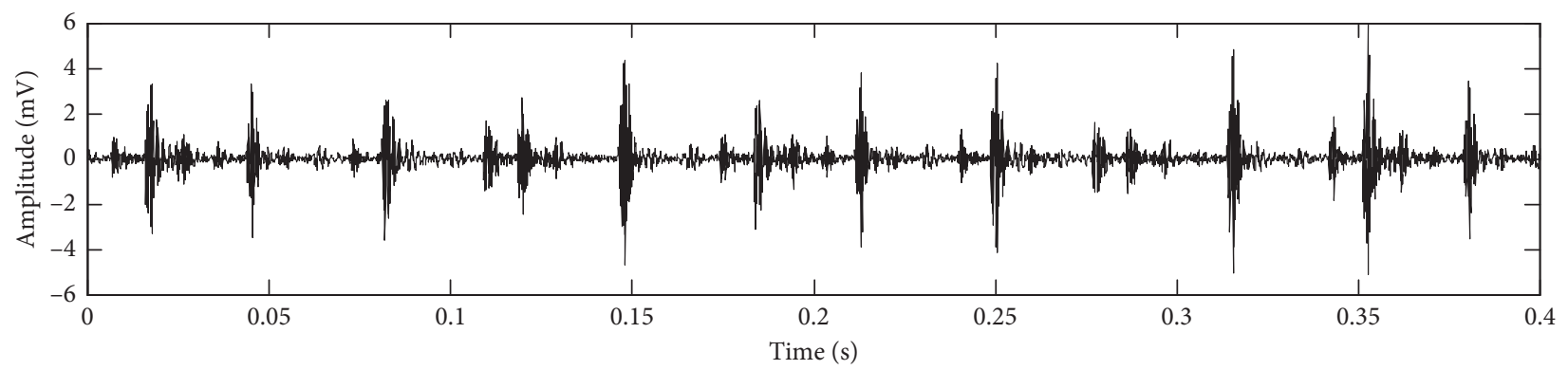

(a)

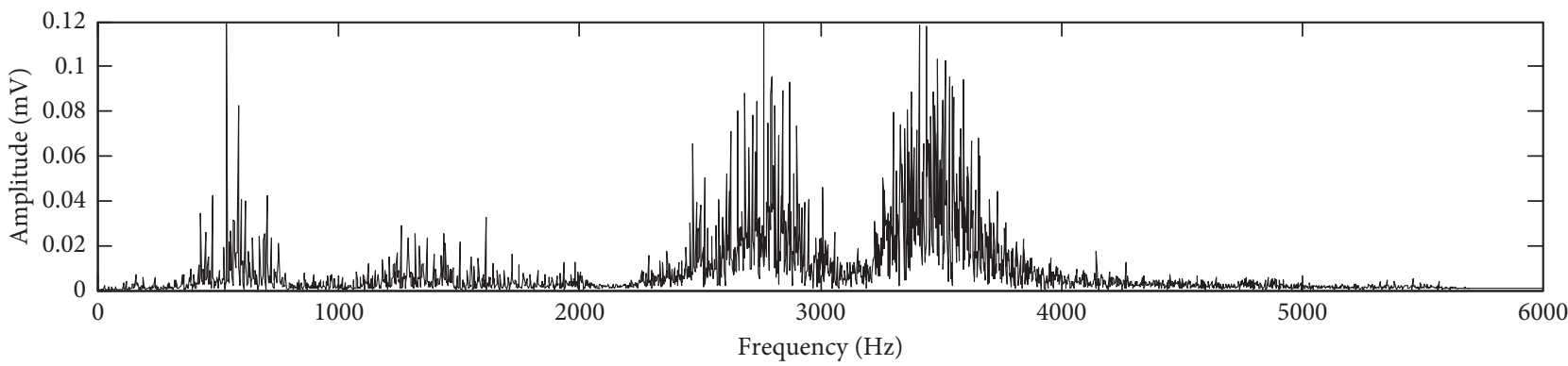

(b)

Figure 9: The outer ring fault signal time domain map and its spectrum.

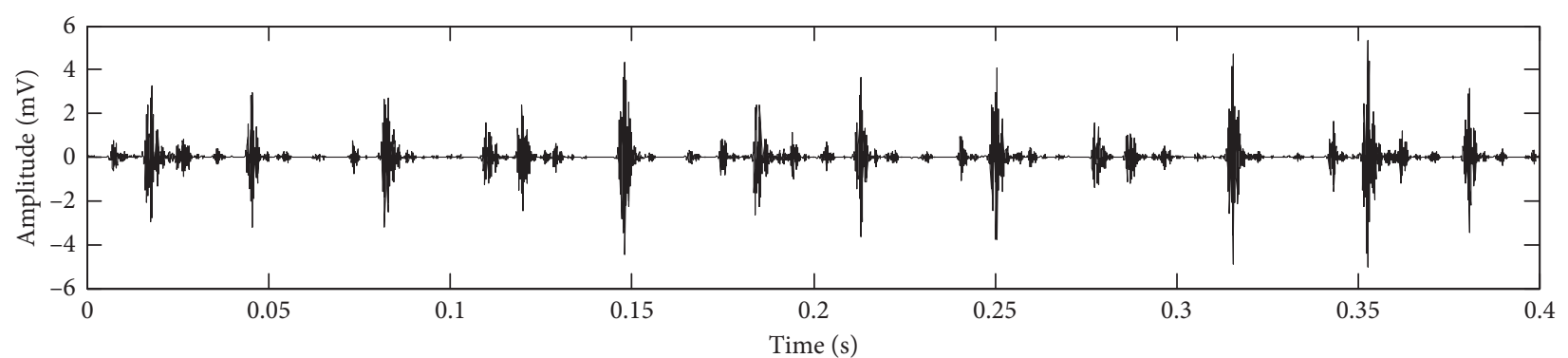

(a)

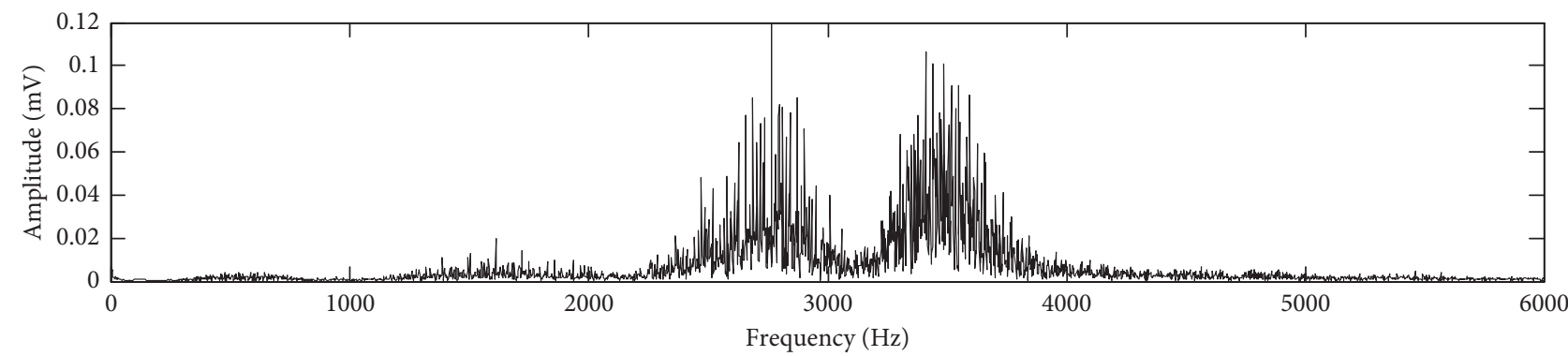

(b)

Figure 10: The outer ring fault signal time domain map and its spectrum after wavelet threshold denoising. 


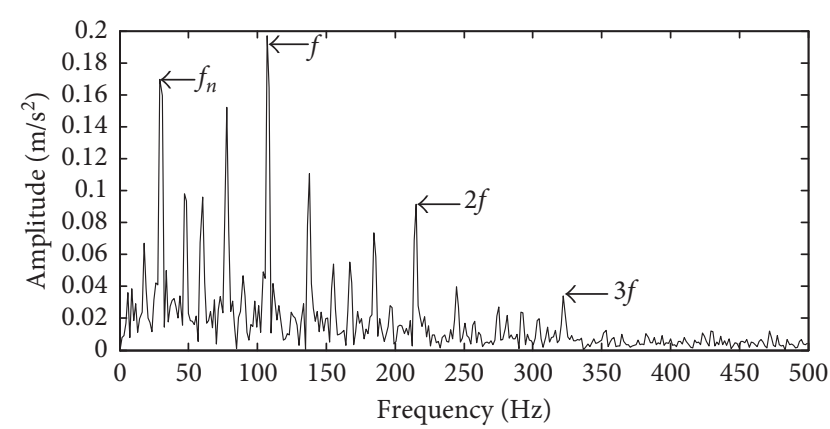

FIGURE 11: Envelope spectrum after NMD of the outer ring fault signal.

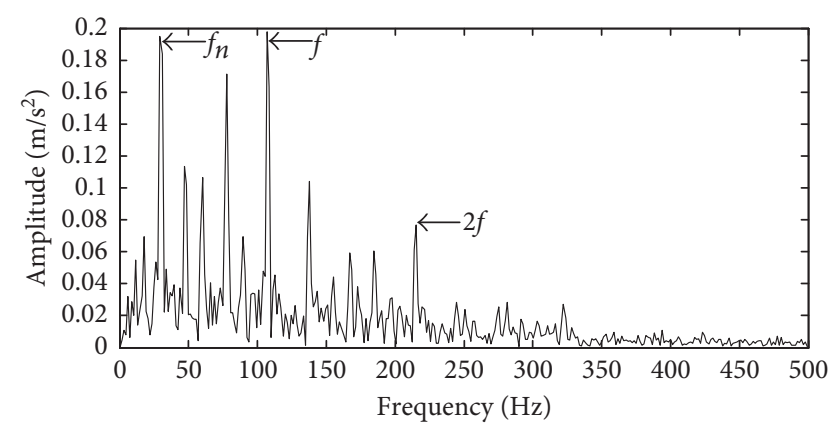

FIGURE 12: Envelope spectrum after EMD of the outer ring fault signal.

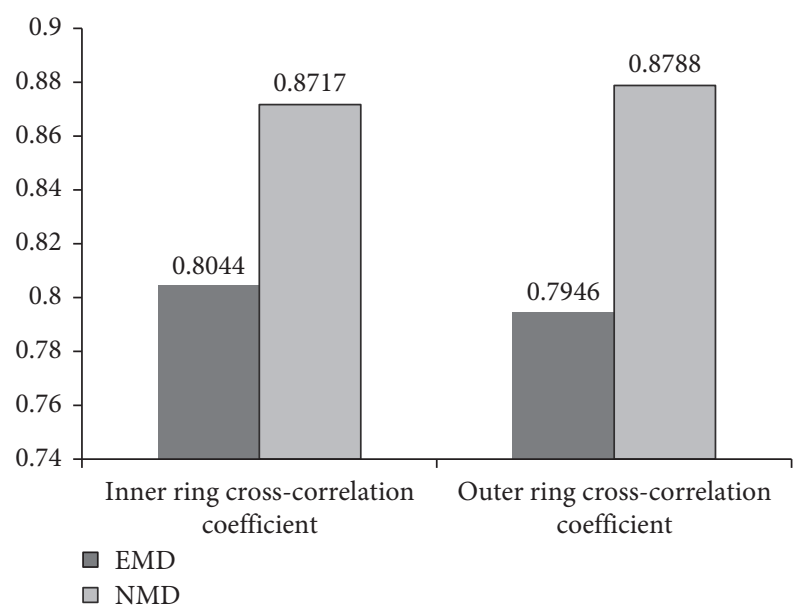

Figure 13: Cross-correlation coefficient comparison chart after NMD and EMD.

cross-correlation coefficient of the inner ring fault signal after the wavelet threshold denoising and EMD is only 0.7946 [28]. The cross-correlation coefficient of the outer ring fault signal after the wavelet threshold denoising and NMD is 0.8717 and the cross-correlation coefficient of the outer ring fault signal after the wavelet threshold denoising and EMD is only 0.8044 [29]. Whether the outer

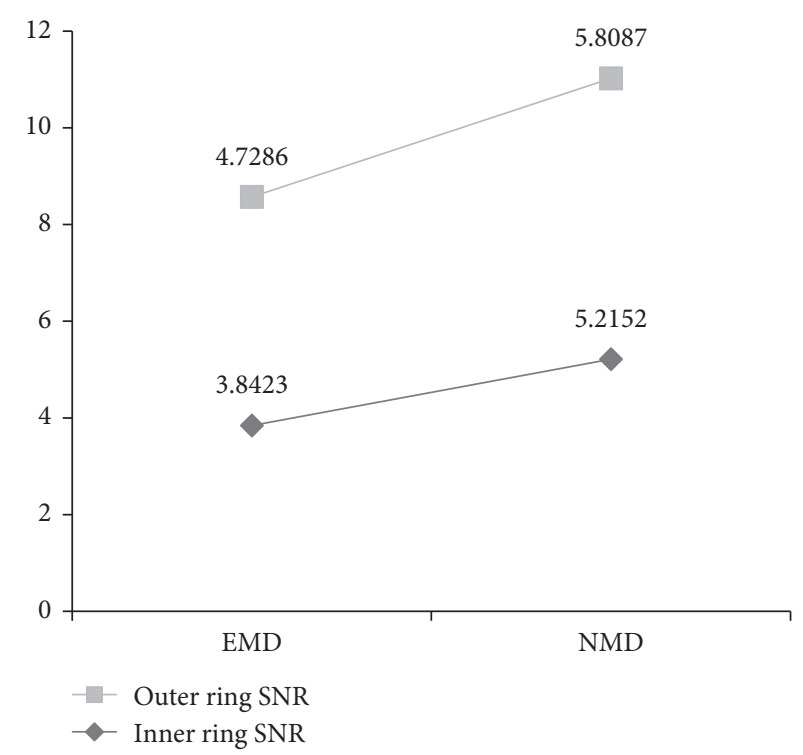

FIGURE 14: SNR comparison chart after NMD and EMD.

ring or the inner ring, the correlation between the NMD decomposed signal and the original signal far exceeds than that of the EMD decomposed signal, which verifies the effectiveness of the method again.

The signal-noise ratio (SNR) [30] and root-mean-square error (RMSE) [31, 32] are calculated, respectively, by formulas (19) and (20):

$$
\begin{aligned}
\mathrm{SNR} & =10 \times \log _{10}\left\{\frac{\sum_{i=1}^{N} S_{i}^{2}}{\sum_{i=1}^{N}\left(S_{i}-S_{i}^{\prime}\right)^{2}}\right\}, \\
\mathrm{RMSE} & =\sqrt{\left(\frac{1}{N} \sum_{i=1}^{N}\left(S_{i}-S_{i}^{\prime}\right)^{2}\right) .}
\end{aligned}
$$

In Figures 14 and 15, both the SNRs of the inner ring and outer ring and the RMSEs of the inner ring and outer ring can be seen, which show that the bearing fault feature extraction methods based on NMD and wavelet threshold denoising are superior to the bearing fault feature extraction method based on EMD and wavelet threshold denoising [33, 34]. The effectiveness of this method is further illustrated.

\section{Conclusion}

This paper studies a fault feature extraction method of rolling bearing based on NMD and wavelet threshold denoising, as well as the fault signals experiment of inner ring and outer ring of rolling bearing. Compared with EMD and wavelet threshold denoising method, this method can effectively extract the accurate fault feature of rolling bearing. As a new method with noise robust and adaptability, NMD has solved the following problems:

(1) The NMD method has the characteristics of selfadaptability and does not require the user to define 


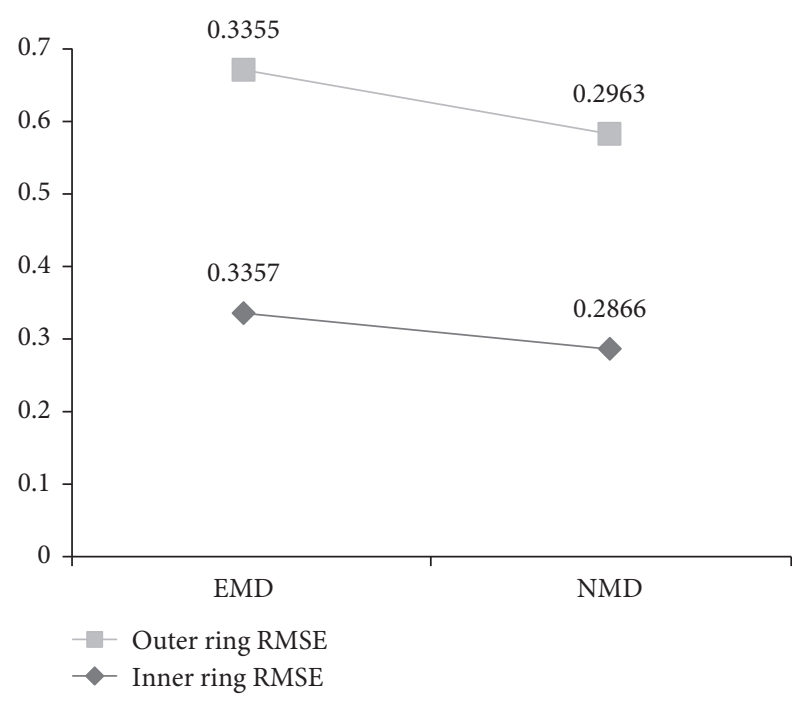

Figure 15: RMSE comparison chart after NMD and EMD.

and set certain parameters specifically, avoiding the problem that the selection of certain parameters is very sensitive.

(2) The NMD method is noise robust and can effectively remove the noise signal from the mixed signal and accurately extract the original signal.

(3) The algorithm of NMD is very efficient, and the original signal after noise removal can be directly obtained, and the original signal does not need to be reconstructed like other methods.

The NMD method overcomes the shortcomings of EMD, such as lacking of strict mathematical foundation, low algorithm efficiency, and modal aliasing. It can be widely used in the field of signal processing and analysis.

\section{Data Availability}

The data used to support the findings of this study are available from the corresponding author upon request.

\section{Conflicts of Interest}

The authors declare that there are no conflicts of interest regarding the publication of this paper.

\section{Acknowledgments}

This research was funded partially by the National Key Research and Development Program of China (2016YFD0701103), Science and Technology Foundation of Outstanding Young Talents of Nanjing Agricultural University (YQ201605), and the Fundamental Research Funds for the Central Universities (KYZ201760).

\section{References}

[1] L. Qian, M. Kang, and X. Q. Fu, "Application of adaptive morphology in bearing fault diagnosis based on VMD,"
Journal of Vibration and Shock, vol. 36, no. 3, pp. 227-233, 2017.

[2] Z. N. Li, M. Zhu, and F. L. Chu, "Mechanical fault diagnosis method based on empirical wavelet transform," Chinese Journal of Scientific Instrument, vol. 35, no. 11, pp. 2423-2432, 2014.

[3] S. T. Wang, J. M. Zhang, and Y. Y. Li, "Rotating machinery fault diagnosis based on mathematical morpology and fuzzy clustering," Chinese Journal of Scientific Instrument, vol. 33, no. 5, pp. 1055-1061, 2012.

[4] X. X. Jiang and S. M. Li, "Adaptive detection on resonance band and using TEO to suppress in-band noise for bearing weak fault diagnosis," Journal of Vibration Engineering \& Technologies, vol. 5, no. 6, pp. 507-521, 2017.

[5] T. Govardhan, A. Choudhury, and D. Paliwal, "Vibration analysis of a rolling element bearing with localized defect under dynamic radial load," Journal of Vibration Engineering \& Technologies, vol. 5, no. 2, pp. 165-175, 2017.

[6] D. Paliwal, A. Choudhury, and T. Govardhan, "Detection of bearing defects from noisy vibration signals using a coupled method of wavelet analysis followed by FFT analysis," Journal of Vibration Engineering \& Technologies, vol. 5, no. 1, pp. 21-34, 2017.

[7] Q. B. Tong, B. Z. Han, and Y. Y. Lin, "A fault feature detection approach for fault diagnosis of rolling element bearings based on redundant second generation wavelet packet transform and local characteristic-scale decomposition," Journal of $\mathrm{Vi}$ bration Engineering \& Technologies, vol. 5, no. 1, pp. 101-110, 2017.

[8] K. Zeki, Y. Ahmet, and B. O. Gurses, "Analysis of rolling element bearing faults via curve length transform," Journal of Vibration Engineering \& Technologies, vol. 2, no. 3, pp. 279298, 2014

[9] Y. Li, M. Xu, X. Liang et al., “Application of Bandwidth EMD and adaptive multiscale morphology analysis for incipient fault diagnosis of rolling bearings," IEEE Transactions on Industrial Electronics, vol. 64, no. 8, pp. 6506-6517, 2017.

[10] Y. Li, M. Xu, Y. Wei et al., "An improvement EMD method based on the optimized rational Hermite interpolation approach and its application to gear fault diagnosis," Measurement, vol. 63, pp. 330-345, 2015.

[11] P. Wang and T. Y. Wang, "Energy weighting method and its application to fault diagnosis of rolling bearing," Journal of Vibroengineering, vol. 19, no. 1, pp. 223-236, 2017.

[12] Z. H. Wu and N. E. Huang, "Ensemble empirical mode decomposition: a noise-assisted data analysis method," $\mathrm{Ad}$ vances in Adaptive Data Analysis, vol. 1, no. 1, pp. 1-41, 2009.

[13] Q. B. Tong, Z. L. Sun, and Z. W. Nie, "Sparse decomposition based on ADMM dictionary learning for fault feature extraction of rolling element bearing," Journal of Vibroengineering, vol. 18, no. 8, pp. 5204-5216, 2016.

[14] H. K. Jiang, Q. S. Cai, and H. W. Zhao, "Rolling bearing fault feature extraction under variable conditions using hybrid order tracking and EEMD," Journal of Vibroengineering, vol. 18, no. 7, pp. 4449-4457, 2016.

[15] D. Iatsenko and P. V. E. McClintock, "Nonlinear mode decomposition: a noise-robust, adaptive decomposition method," Physical Review E, vol. 92, no. 3, 2015.

[16] J. M. Li, M. Li, and J. F. Zhang, "Rolling bearing fault diagnosis based on time-delayed feedback monostable stochastic resonance and adaptive minimum entropy deconvolution," Journal of Sound and Vibration, vol. 401, pp. 139-151, 2017.

[17] A. Issam, F. Nadir, and B. Nadir, "A new time-frequency method for identification and classification of ball bearing 
faults," Journal of Sound and Vibration, vol. 397, pp. 241-265, 2017.

[18] L. L. Cui, X. Y. Gong, and J. Y. Zhang, "Double-dictionary matching pursuit for fault extent evaluation of rolling bearing based on the Lempel-Ziv complexity," Journal of Sound and Vibration, vol. 385, pp. 372-388, 2016.

[19] Z. Matej, Z. Samo, and P. Ivan, "EEMD-based multiscale ICA method for slewing bearing fault detection and diagnosis," Journal of Sound and Vibration, vol. 370, pp. 394-423, 2016.

[20] G. L. He, K. Ding, and H. B. Lin, "Fault feature extraction of rolling element bearings using sparse representation," Journal of Sound and Vibration, vol. 366, pp. 514-527, 2016.

[21] Y. B. Li, M. Q. Xu, and R. X. Wang, "A fault diagnosis scheme for rolling bearing based on local mean decomposition and improved multiscale fuzzy entropy," Journal of Sound and Vibration, vol. 360, pp. 277-299, 2016.

[22] X. L. Liu, H. G. Liu, and J. H. Yang, "Improving the bearing fault diagnosis efficiency by the adaptive stochastic resonance in a new nonlinear system," Mechanical Systems and Signal Processing, vol. 96, pp. 58-76, 2017.

[23] J. X. Ma, F. Y. Xu, and K. Huang, "GNAR-GARCH model and its application in feature extraction for rolling bearing fault diagnosis," Mechanical Systems and Signal Processing, vol. 93, pp. 175-203, 2017.

[24] M. Zhang, Z. N. Jiang, and K. Feng, "Research on variational mode decomposition in rolling bearings fault diagnosis of the multistage centrifugal pump," Mechanical Systems and Signal Processing, vol. 93, pp. 460-493, 2017.

[25] Y. B. Li, X. H. Liang, and M. Q. Xu, "Early fault feature extraction of rolling bearing based on ICD and tunable Q-factor wavelet transform," Mechanical Systems and Signal Processing, vol. 86, pp. 204-223, 2017.

[26] C. L. Li, F. R. Kong, and W. G. Huang, "Rolling bearing fault diagnosis based on EEMD and Laplace wavelet," Journal of Vibration and Shock, vol. 33, no. 3, pp. 63-69, 2014.

[27] S. M. Li and X. L. Li, Modern Analysis Techniques and Application of Vibration Signal, National Defend Industry Press, Beijing, China, 2008.

[28] Z. Meng and S. S. Li, "Rolling bearing fault diagnosis based on improved wavelet threshold de-noising method and HHT," Journal of Vibration and Shock, vol. 14, pp. 204-208, 2013.

[29] H. C. Wang and F. Hao, "Fault diagnosis of rolling element bearing based on wavelet kernel principle component analysis-coupled hidden Markov model," Journal of Vibroengineering, vol. 19, no. 8, pp. 5992-6006, 2017.

[30] C. X. Wu, T. F. Chen, and R. Jiang, "Bearing fault diagnosis via kernel matrix construction based support vector machine," Journal of Vibroengineering, vol. 19, no. 5, pp. 3445-3461, 2017.

[31] H. G. Liu, S. Han, and J. H. Yang, "Improving the weak feature extraction by adaptive stochastic resonance in cascaded piecewise-linear system and its application in bearing fault detection," Journal of Vibroengineering, vol. 19, no. 4, pp. 2506-2520, 2017.

[32] X. M. Xu, Y. P. Jiang, and H. P. Lee, "Multi-objective optimal design of sandwich panels using a genetic algorithm," Engineering Optimization, vol. 49, no. 10, pp. 1665-1684, 2017.

[33] H. K. Jiang, F. Wang, and H. D. Shao, "Rolling bearing fault identification using multilayer deep learning convolutional neural network," Journal of Vibroengineering, vol. 19, no. 1, pp. 138-149, 2017.

[34] X. M. Xu, Y. P. Jiang, N. Chen, and H. P. Lee, "Dynamic behavior of a vehicle with rear axle compliance steering,"
Journal of Vibroengineering, vol. 18, no. 4, pp. 4483-4497, 2017. 


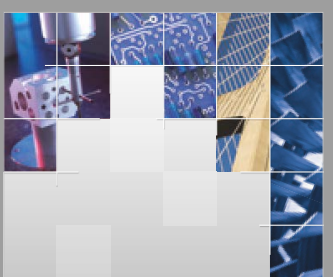

\section{Enfincering}
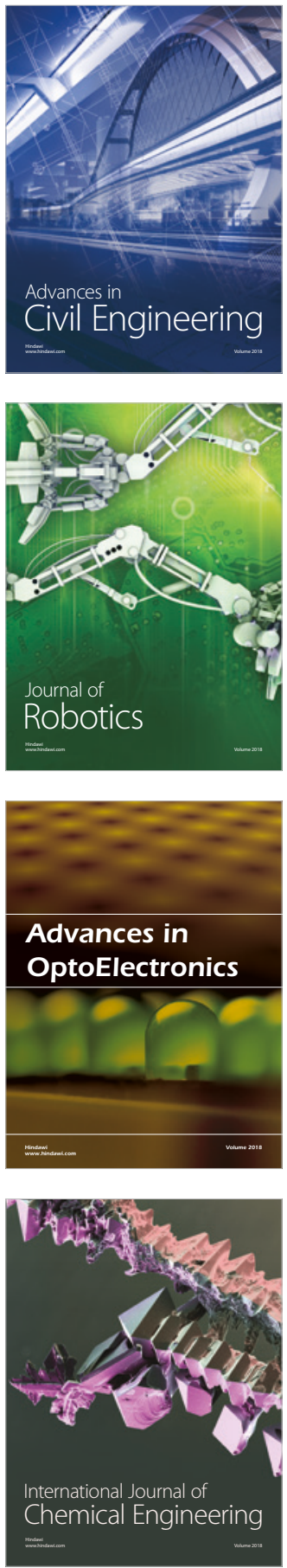

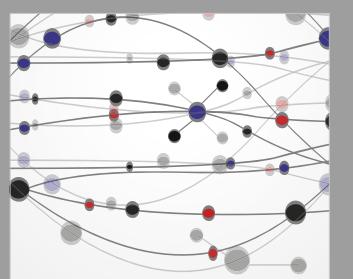

\section{Rotating \\ Machinery}

The Scientific World Journal

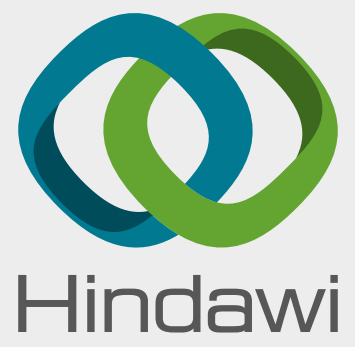

Submit your manuscripts at

www.hindawi.com
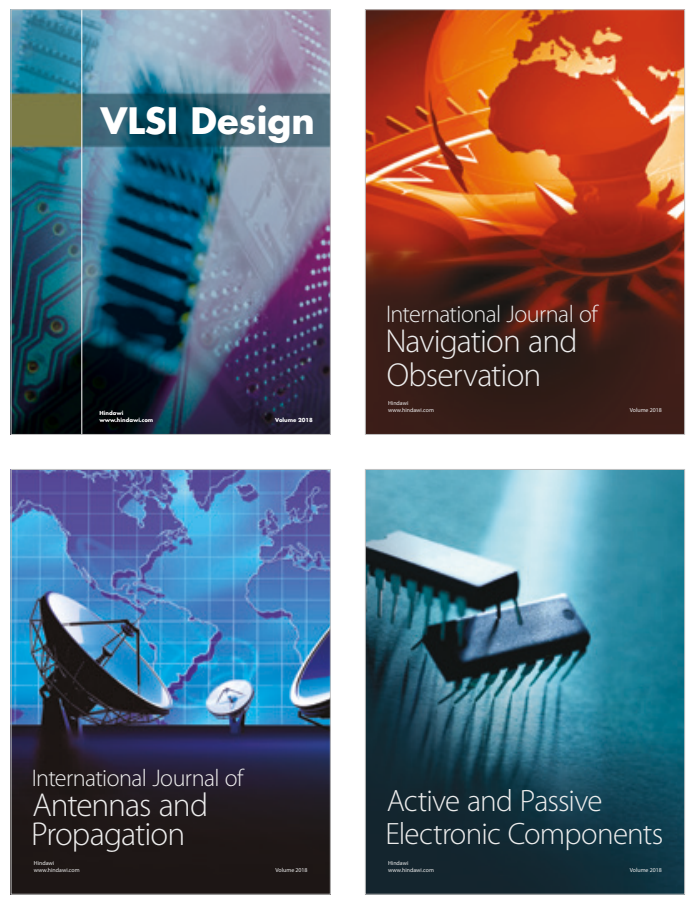
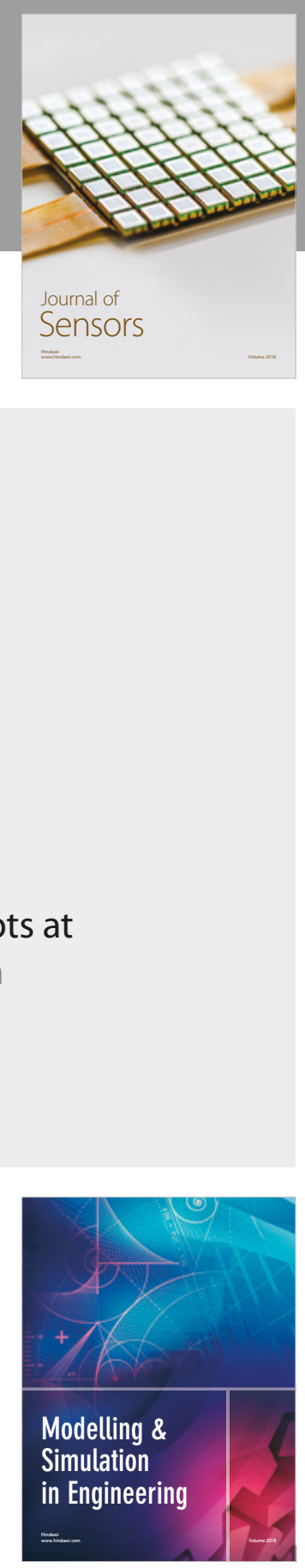

\section{Advances \\ Multimedia}
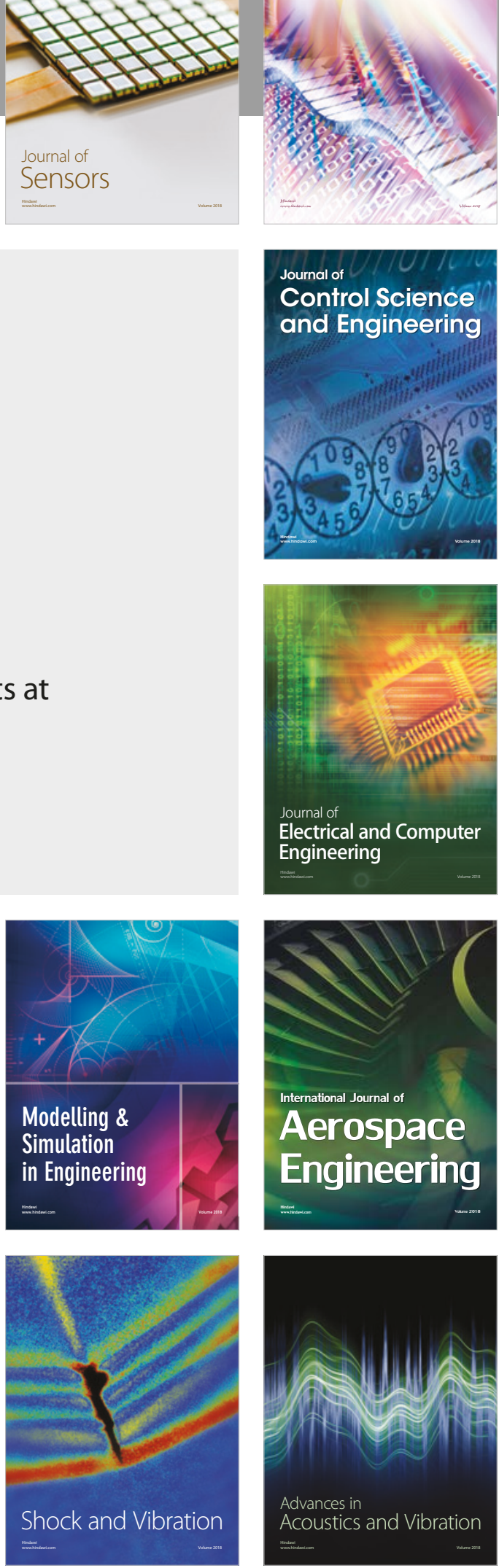